Garner and Theriot, 2020

\title{
Leading edge stability in motile cells is an emergent property of branched actin network growth
}

\author{
Rikki M. Garner ${ }^{1,2}$ and Julie A. Theriot ${ }^{2 *}$ \\ ${ }^{1}$ Biophysics Program, Stanford University School of Medicine, Stanford, CA, USA \\ ${ }^{2}$ Department of Biology and Howard Hughes Medical Institute, University of Washington, Seattle, WA, USA \\ *Correspondence to: jtheriot@uw.edu
}

One sentence summary: An experimental and computational investigation of fluctuation dynamics at the leading edge of motile cells demonstrates that the specific angular geometry of Arp2/3-mediated actin network branch formation lies at the core of a successful biological noise-suppression strategy. 
Garner and Theriot, 2020

1 Abstract: Animal cell migration is predominantly driven by the coordinated, yet stochastic,

2 polymerization of thousands of nanometer-scale actin filaments across micron-scale cell leading edges. It

3 remains unclear how such inherently noisy processes generate robust cellular behavior. We employed

4 high-speed, high-resolution imaging of migrating neutrophil-like HL-60 cells to explore the fine-scale

5 dynamic shape fluctuations that emerge and relax throughout the process of leading edge maintenance.

6 We then developed a minimal stochastic model of the leading edge that is able to reproduce this stable

7 relaxation behavior. Remarkably, we find that lamellipodial stability naturally emerges from the interplay

8 between branched actin network growth and leading edge shape - with no additional feedback required -

9 based on a synergy between membrane-proximal branching and lateral spreading of filaments. These

10 results thus demonstrate a novel biological noise-suppression mechanism based entirely on system

11 geometry. Furthermore, our model suggests that the Arp2/3-mediated $\sim 70-80^{\circ}$ branching angle optimally

12 smooths lamellipodial shape, addressing its long-mysterious conservation from protists to mammals. 
Main text:

14 Cell migration driven by actin polymerization plays an essential role in countless organisms spanning the

eukaryotic tree of life $(1,2)$. Across this broad phylogeny, cells have been observed to form a dizzying array of protrusive actin structures, each exhibiting unique physical and biological properties (3). In all cases, the fundamental molecular unit of these micron-scale structures is the single actin filament, which polymerizes stochastically by addition of single monomers to push the leading edge membrane forward (4-7). Higher-order actin structures, and the biological functions they robustly enable, are therefore mediated by the collective action of thousands of stochastically growing filaments (8). It remains an open question how cells control for - or leverage - this inherent stochasticity to maintain stable leading edge protrusions over length and time scales more than three orders of magnitude larger than the scales of actin monomer addition $(9,10)$.

Perhaps the archetype of dynamically stable actin structures is the lamellipodium, a flat "leaf-like" protrusion that is $\sim 200 \mathrm{~nm}$ tall, up to $100 \mu \mathrm{m}$ wide, and filled with a dense network of dendritically branched actin filaments (11-14). Cell types that undergo lamellipodial migration (most notably fish epidermal keratocytes and vertebrate neutrophils) can maintain a single, stable lamellipodium for minutes to hours, allowing the cells to carry out their biological functions $(15,16)$. For example, in their in vivo role as first responders of the innate immune system, neutrophils must undergo persistent migration over millimeter-scale distances to reach sites of inflammation and infection $(17,18)$. Regardless of the cell type, the origins of this striking stability in the face of stochastic actin filament polymerization remain elusive. The stability of lamellipodia has been theoretically proposed to depend on the dendritically branched structure of their actin networks, wherein filaments are oriented at an angle relative to the local membrane normal, allowing growing filament tips to spread out laterally along the leading edge as they polymerize $(16,19)$. (Although any acute angle would permit spreading, we note that filament orientation in cells has been experimentally observed to be highly stereotyped, averaging $\pm 35^{\circ}$ relative to the 
membrane normal $(20,21)$ - approximately one half of the highly evolutionarily-conserved $\sim 70^{\circ}$ branch

angle mediated by the Arp2/3 complex (22-24).) Experimental efforts have further identified a number of

additional potential contributors to lamellipodial stability, including membrane tension (25-29), plasma membrane curvature-sensing proteins (29), and force-feedback via directional filament branching (30). Seeking to dissect the origins of lamellipodial stability, we pursued complimentary experimental and computational methodologies. First, we performed high-speed, high-resolution microscopy on migrating human neutrophil-like HL-60 cells to monitor their leading edge shape dynamics. In contrast to the remarkable overall lamellipodial stability observed over minutes, high-speed imaging revealed that the leading edge shape is extremely dynamic at shorter time and length scales, constantly undergoing finescale fluctuations around the average cell shape. We determined that these shape fluctuations continually dissipate (thereby enabling long time scale lamellipodial maintenance) in a manner quantitatively consistent with viscous relaxation back to the time-averaged leading edge shape. We next developed a minimal stochastic model of branched actin network growth against a flexible membrane, broadly applicable to a wide variety of cell types, that was able to recapitulate the global leading edge stability and

fine-scale fluctuation relaxation behavior observed in cells. Our model suggests that the suppression of stochastic fluctuations is an intrinsic, emergent property of collective actin dynamics at the leading edge, as branched network geometry alone is necessary and sufficient to generate lamellipodial stability. Moreover, we find that the evolutionarily-conserved geometry, the $\sim 70^{\circ}$ branching angle of the Arp $2 / 3$ complex, optimally quells shape fluctuations.

Fine-scale leading edge shape fluctuations revealed at high spatiotemporal resolution

58 Neutrophils form lamellipodia that are intrinsically lamellar, maintaining a thin, locally flat sheet of actin 
Garner and Theriot, 2020

neutrophil-like HL-60 cells (31) within quasi-two-dimensional confinement between a glass coverslip and an agarose pad overlay (32). In addition to serving as an excellent in vitro model for neutrophil surveillance of tissues, this assay allows for easy visualization and quantification of lamellipodial dynamics by restraining the lamellipodium to a single imaging plane. Cells in this type of confinement can migrate persistently, maintaining nearly-constant cell shape, for time scales on the order of minutes to hours $(15,33)$. In order to capture leading edge dynamics on time scales more relevant to the stochastic growth of individual filaments, we performed high-speed $(20 \mathrm{~Hz})$ imaging of migrating HL-60 cells. These experiments revealed dynamic, fine-scale fluctuations around the average leading edge shape (Fig. 1A, Movie S1, Fig. S1-S2, Materials and Methods), reminiscent of an excitable system in which local instabilities in the leading edge emerge, grow, and then relax. Consistent with the idea that these fluctuations arise from stochasticity in actin growth, kymograph analysis of curvature and velocity (Fig. 1B-C) showed that relatively long-lived curvature fluctuations are formed by the continual time-integration of very short-lived (sub-second, sub-micron) velocity fluctuations. Because the average cell shape remains constant over time, there must be some form of feedback acting on leading edge curvature to sustain stable lamellipodial growth. These rich, measurable fine-scale dynamics therefore provide a unique opportunity to directly observe the time-evolution of leading edge maintenance. Taking advantage of our high-precision measurements, we aimed to quantitatively investigate the properties of the observed fluctuations, with the goal of determining the mechanisms by which molecular machinery at the leading edge coordinates the stochastic polymerization of individual actin filaments.

\section{Lamellipodial stability mediated by viscous relaxation of shape fluctuations}

The relaxation of fine-scale shape fluctuations back to the steady-state leading edge shape is essential for the long time scale stability of lamellipodia. As for any physical system, the nature of this relaxation 
Garner and Theriot, 2020

A

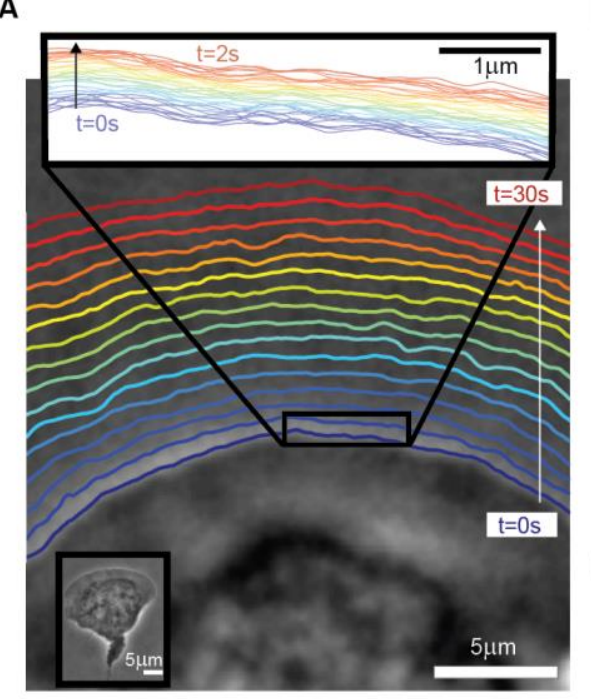

E

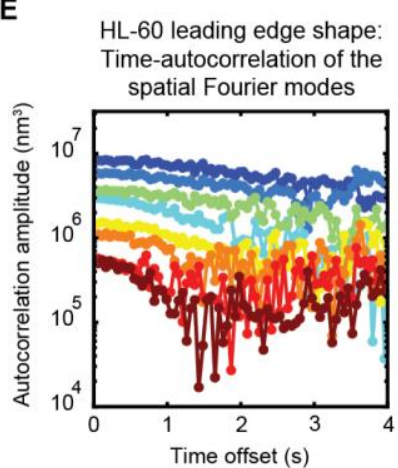

$\mathbf{F}$
B

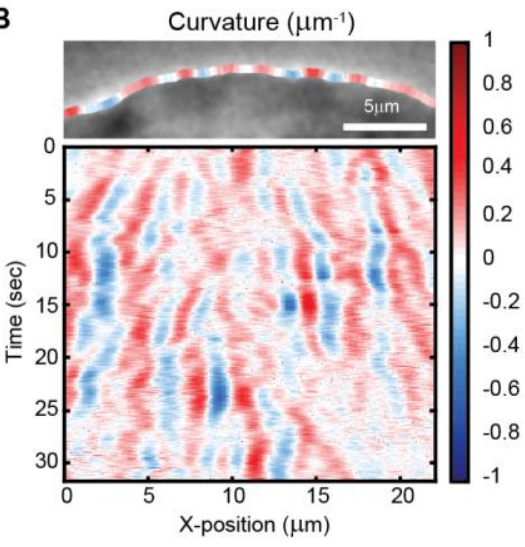

D

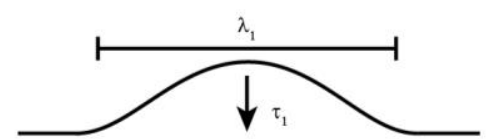

c
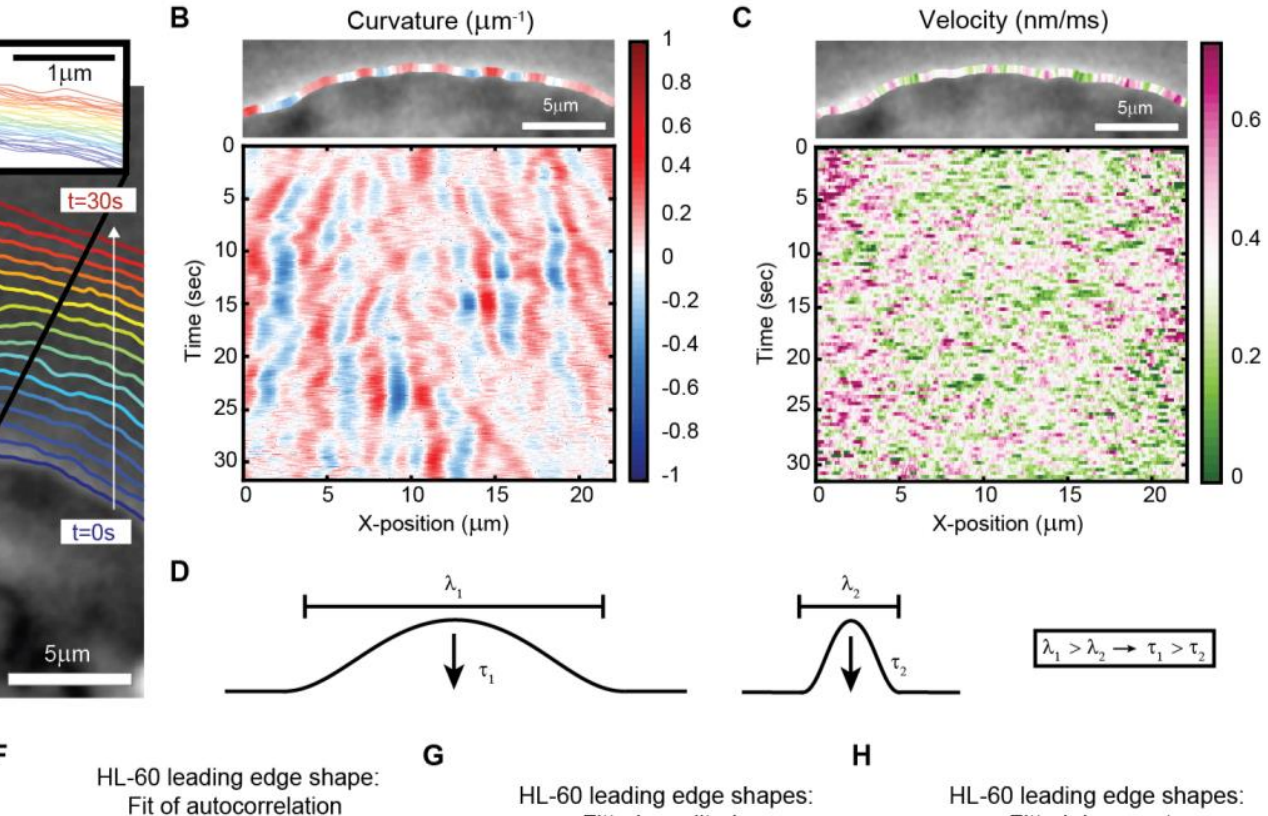
Fit of autocorrelation decay curves to $\mathrm{Ae}^{-1 t}$

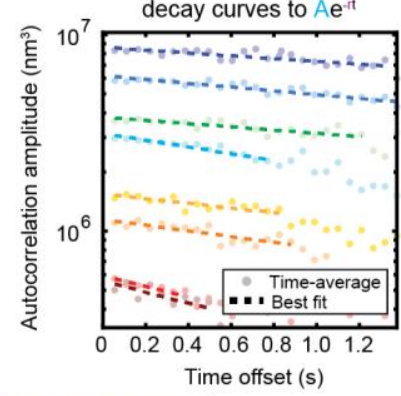

G

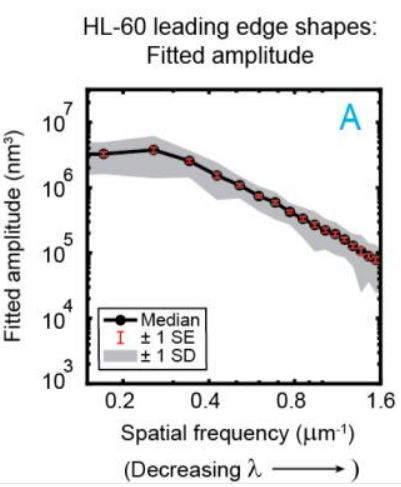

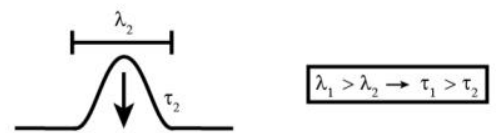

H
HL-60 leading edge shapes: Fitted decay rate

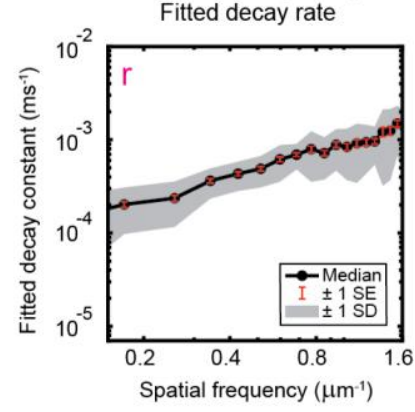

Fig. 1. High-speed, high-resolution imaging reveals fine-scale fluctuations in leading edge shape. (A-C) Example of leading edge fluctuations extracted from a representative migrating HL-60 cell. (A) Phase contrast microscopy image from the first frame of a movie, overlaid with segmented leading edge shapes from time points increasing from blue to red in $2 \mathrm{sec}$ intervals. Top Inset: Magnification of the segmented leading edge between $t=0-2 \mathrm{sec}$ increasing from blue to red in $50 \mathrm{~ms}$ intervals. Bottom Inset: A de-magnified image of the whole cell at the last time point. (B-C) Kymographs of curvature (B) and velocity (C). (D) Schematic demonstrating a commonly observed trend between fluctuation wavelength and relaxation time. (E) Autocorrelation amplitude (complex magnitude) of the spatial Fourier transform plotted as a function of time offset from a representative video of a cell. (F) Best fit of the autocorrelation data shown in (E) to an exponential decay, fitted out to a drop in amplitude of 2/e. (G-H) Fitted parameters of the autocorrelation averaged over 67 cells.

83 reflects the system's underlying physical properties; in this case, the characteristics of - and interactions

84 between - actin filaments and the membrane. To provide a framework for exploration of the physical

85 mechanisms underlying stable lamellipodial protrusion, we quantified the relaxation dynamics by 
Garner and Theriot, 2020

87 this context, this analytical technique calculates the extent to which the lamellipodium contour loses

88 similarity with the shape at previous time points as fluctuations emerge and relax. As most material

89 systems (actively-driven or otherwise) exhibit relaxation behavior with a characteristic wavelength-

90 dependence (e.g., Fig. 1D), we performed Fourier decomposition on the leading edge shape to separate

91 out fluctuations at different length scales, and then performed autocorrelation analysis separately on each

92 Fourier mode.

This analysis revealed a monotonic relaxation of shape fluctuations at each wavelength

94 (Fig. 1E-F); the decay is well-fit by an exponential form (Fig. 1F), consistent with overdamped viscous relaxation. A clear wavelength-dependence is observed, with shorter wavelengths decaying faster and

96 having smaller amplitudes (Fig. 1G-H). This general trend is shared by many physical systems with linear 97 elastic constraints, such as idealized membranes (34) and polymers (35) freely fluctuating under Brownian 98 motion, but can be contrasted with systems that have a dominant wavelength, as in the case of buckling 99 or wrinkling of materials under compression (36).

\section{Leading edge stability as an emergent property of branched actin growth}

103 comparison with physical models. In order to determine the biophysical mechanisms underlying the 104 observed leading edge dynamics, we aimed to reproduce this behavior in a stochastic model of branched 105 actin network growth against a membrane (Fig. 2A-C, Movie S2, Materials and Methods). Previous 106 stochastic models of protrusive actin-based forces largely focused on actin polymerization against rigid 107 obstacles (e.g., the bacterial cell wall for the Listeria comet tail $(37,38)$ or a single, flat membrane segment 108 in models of lamellipodia (27)). We expanded on this general framework to incorporate a two-dimensional 109 leading edge with filaments polymerizing against a flexible membrane, which is modeled as a system of 
Garner and Theriot, 2020

A

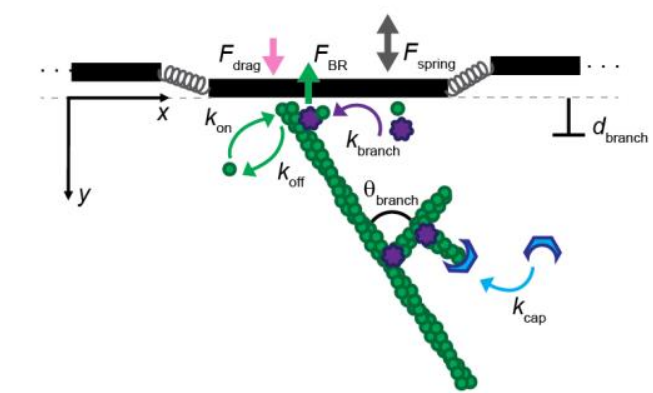

D

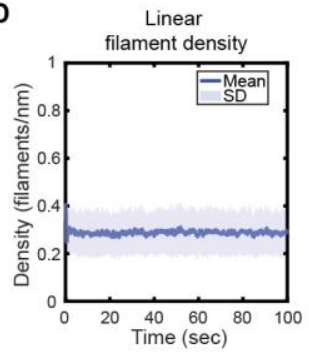

I

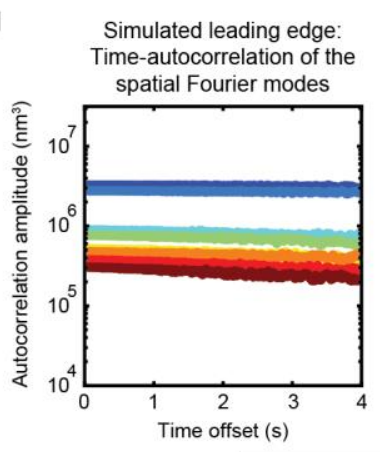

$(\mathbf{I} \boldsymbol{)})$ shortest $\lambda \longrightarrow$ longest $\lambda$
B

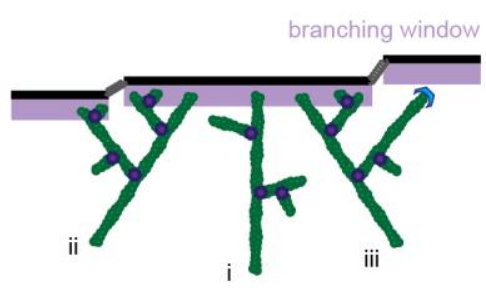

C

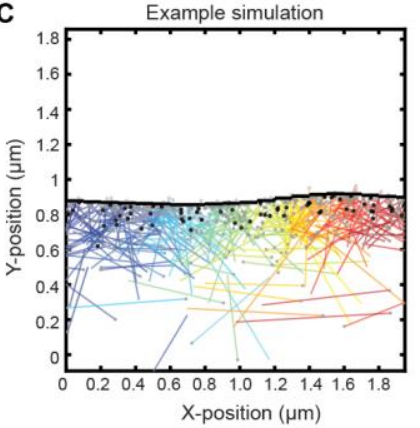

G
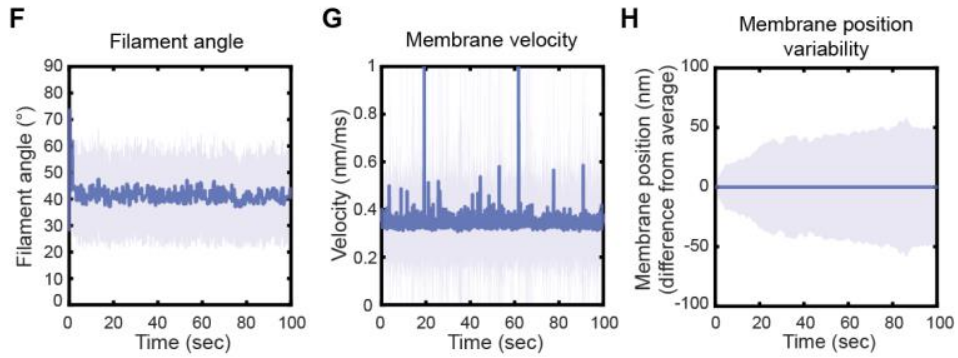

K
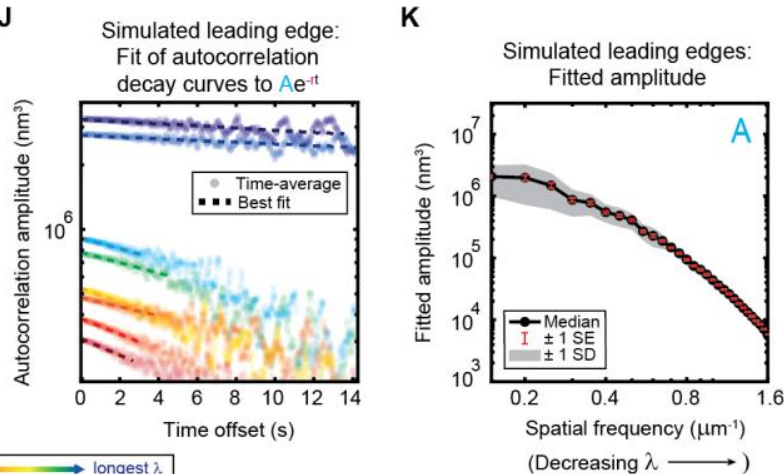

L

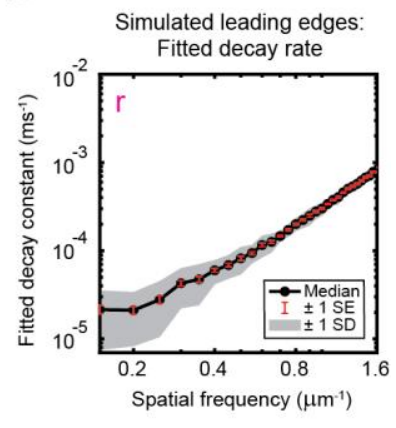

Fig. 2. Minimal model of branched actin growth recapitulates leading edge stability and shape fluctuation relaxation. (A-B) Model schematic. Black lines, membrane; green circles, actin; purple flowers, Arp2/3 complex; blue crescents, capping protein. Rates: $k_{\text {on }}$, polymerization; $k_{\text {off, }}$

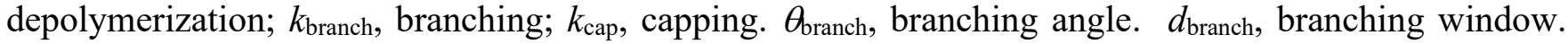

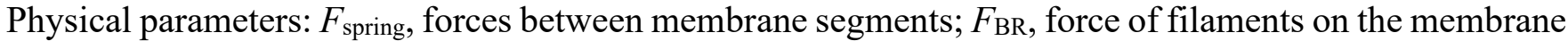

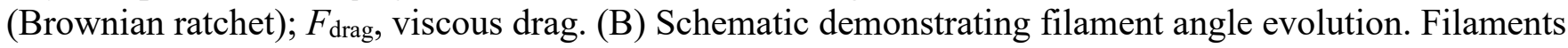
growing perpendicular to the leading edge (i) outcompete their progeny (branches), leading to a reduction in filament density; filaments growing at an angle (ii and iii) make successful progeny. Filaments spreading down a membrane positional gradient (ii) are more evolutionarily successful than those spreading up (iii). (C) Simulation snapshot: Black lines, membrane; colored lines, filament equilibrium position and shape; gray dots, barbed ends; black dots, capped ends; filament color, x-position of membrane segment filament is pushing (increasing across the $\mathrm{x}$-axis from blue to red). (D-H) For a representative simulation, mean (solid line) and standard deviation (shading) of various membrane and actin filament properties as a function of simulation time. Note for linear filament density (D) lamellipodia are $\sim 10$ filament stacks tall along the z-axis, giving mean filament spacing of 10/density $\sim 30 \mathrm{~nm}$. (I-L) Autocorrelation analysis and fitting for a representative simulation (I-J) as well as best fit parameters averaged over 40 simulations (K-L). 
Garner and Theriot, 2020

111

112

113

114

115

116

117

118

119

120

121 "daughter" actin filaments a

122

123

124

125

126

127

128

129

130

131

132 (with respect to the membrane normal), $\sim 0.35 \mathrm{~nm} / \mathrm{ms}$ for membrane velocity, and $\sim 50 \mathrm{~nm}$ for membrane

133 fluctuation amplitude $(11,20,21)$. 
Garner and Theriot, 2020

135 by an exponential decay of shape fluctuations (Fig. 2I-J). Furthermore, the minimal model correctly 136 predicts the monotonic trends of fluctuation amplitude and decay time scale with wavelength (Fig. 2K-L) 137 in a way that was not sensitive to our choices of simulation time step, membrane segment length, and 138 overall length of the leading edge (Fig. S3-5). It should be noted that the generation of the simulated data 139 in Figure 2K-L did not involve any curve-fitting (and therefore no free parameters that could be fit) to the 140 experimentally-measured autocorrelation dynamics. The simulated fluctuation relaxation behavior, 141 qualitatively reproducing our experimental measurements, emerges directly from the molecular-scale 142 actin growth model, in which all biochemical parameters were estimated from measurements in the 143 existing literature (Table S1-S2) - leaving no free simulation parameters.

\section{Predicting effects of drug treatment with Latrunculin B}

146 We were interested in further assaying the predictive power of this minimal stochastic model. As an example, we elected to test whether the model could correctly predict the response of HL-60 cells to 148 treatment with the drug Latrunculin B, which binds to and sequesters actin monomers. Qualitatively, cells treated with Latrunculin B (Movie S3) present with enhanced bleb formation and more variable leading

150 edge shapes, in comparison with cells treated with a DMSO vehicle control (Movie S4). In our model, 151 addition of this drug can be simulated by reducing the free monomer concentration, which consequently 152 reduces both the polymerization rate and the branching rate. At low effective doses, subtle but measurable 153 changes to leading edge fluctuations were predicted: specifically, an increase in the amplitudes at large 154 wavelengths, and a decrease in the decay rates across all wavelengths (Fig 3A-B). Our experimental 155 results were consistent with these quantiative predictions; Latrunculin B-treated cells exhibited increased 156 fluctuation amplitudes and decreased fluctuation rates over the predicted ranges (Fig. 3C-D). 
Garner and Theriot, 2020

Biochemical perturbation: reduction in free monomer concentration
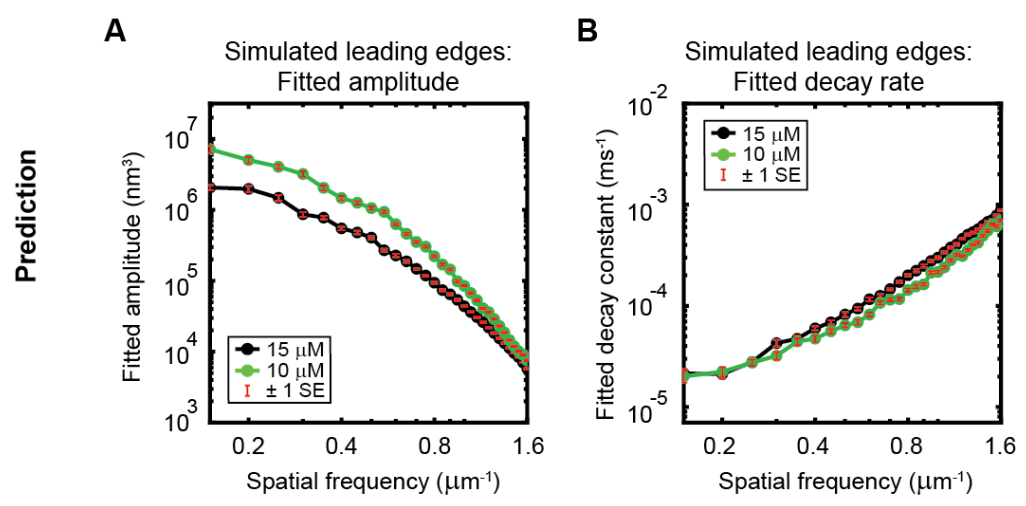

Fig. 3. Minimal model correctly predicts response of $\mathrm{HL}-60$ cells to drug treatment. Predicted and experimentally-measured response of the autocorrelation decay fit parameters to drug treatment with Latrunculin B, plotted as in Fig. 1G-H. (A-B) Predicted response to a reduction in the free monomer concentration (green, $10 \mu \mathrm{M}$ )
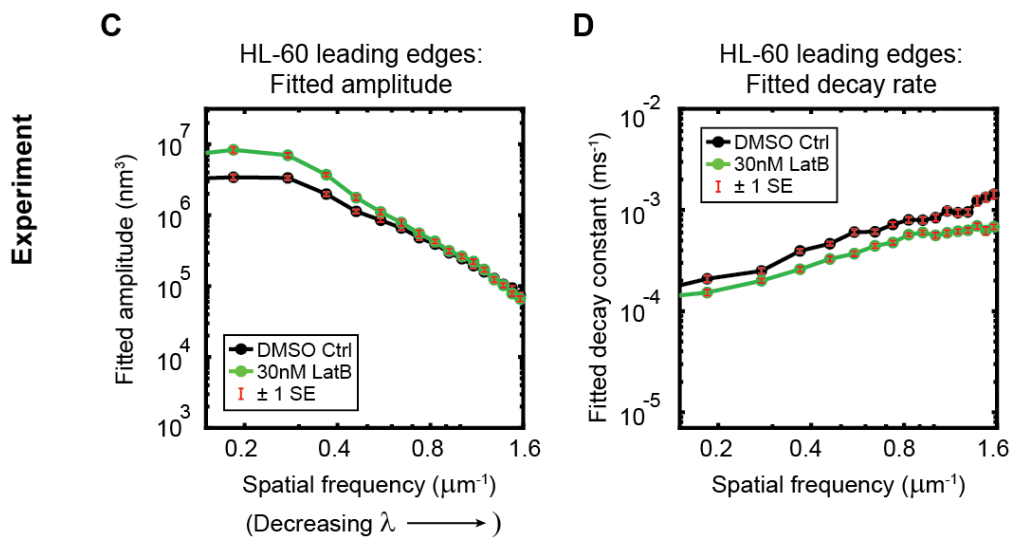
compared to the standard concentration used in this work (black, $15 \mu \mathrm{M}$ ) medians over 40 simulations for each condition. (C-D) Experimentally measured behavior: DMSO control medians over 67 cells (same data as plotted in Fig. 1G-H). 30nM Latrunculin $\mathrm{B}-$ medians over 34 cells.

\section{Geometry as the core determinant of simulated leading edge stability}

Given the success of the model in reproducing experimental results, we next wanted to determine which features of the simulation were responsible for leading edge stability and relaxation of fluctuations. The simplicity of the model allowed us to determine the stability mechanism by process of elimination, selectively removing elements of the model (Materials and Methods) and determining whether stability was retained. Given that leading edge protrusion is resisted by a flexible membrane, we initially expected that shape fluctuations would be dominated by membrane tension or membrane bending rigidity. To assay the importance of these membrane properties, we removed the forces between the membrane segments (Fig. 2A, $F_{\text {spring }}$ ) from the simulation (Materials and Methods). Surprisingly, the coupling between the membrane segments (i.e., the effects of tension and bending at length scales larger than the size of an individual membrane segment) was completely dispensable for leading edge stability (Fig. 4A-E). 
Garner and Theriot, 2020
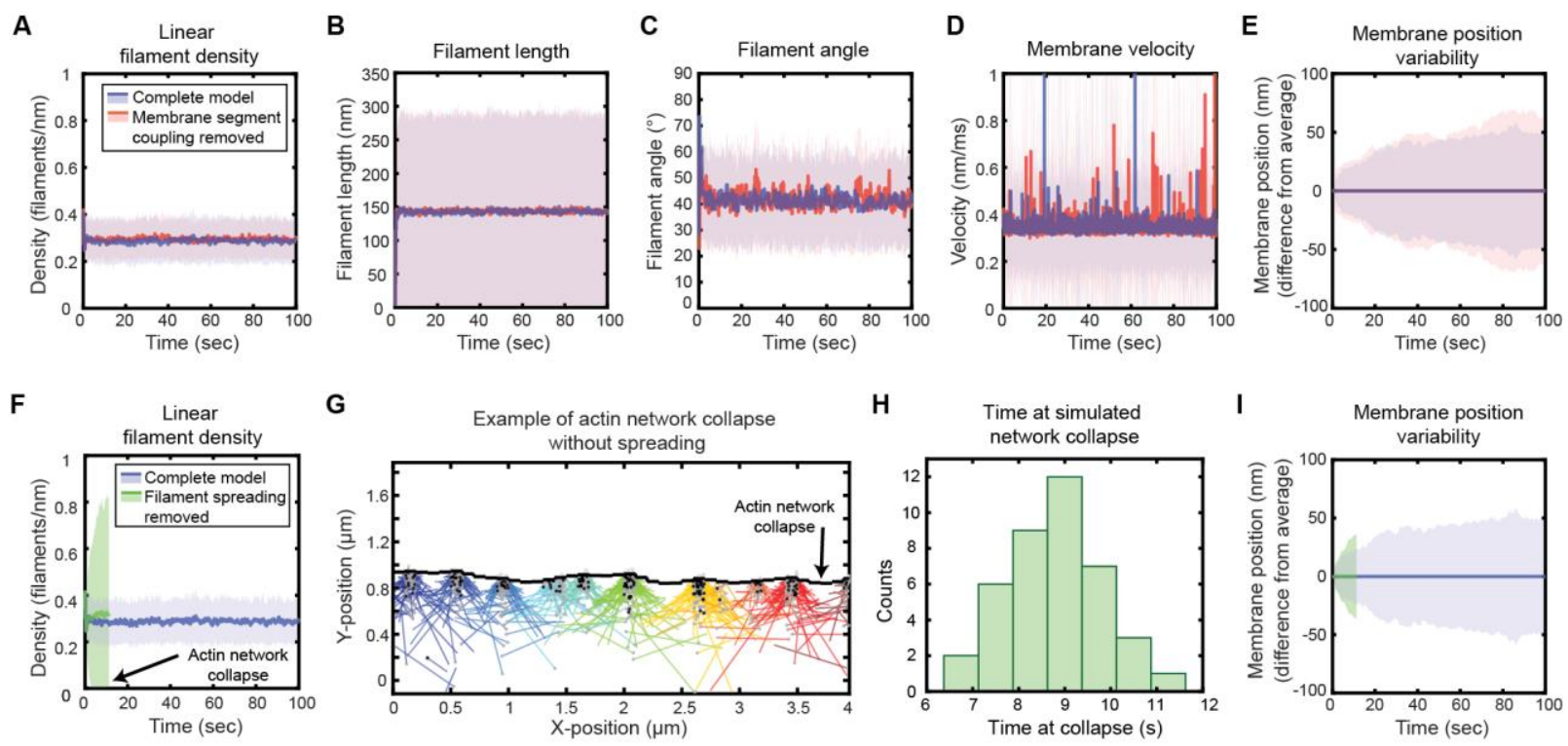

$\mathbf{J}$
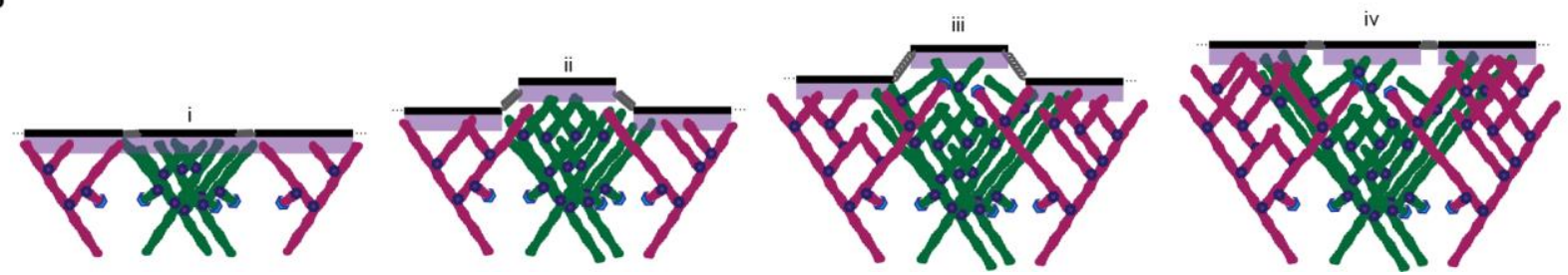

Fig. 4. Simulated lamellipodial stability is governed by leading edge geometry. (A-E) Comparison of leading edge properties with and without the coupling of the membrane segments by tension and bending rigidity (no coupling: $F_{\text {spring }}=0$ in Fig. 2A), plotted as in Fig. 2D-H. (F-I) Comparison of leading edge properties with and without the ability of filaments to spread between neighboring membrane segments. (G) A snapshot of the simulation after filament network collapse (defined as a state where at least $25 \%$ of the membrane segments have no associated filaments). (F,G,I) Plots made from the same simulation. (H) A histogram of the average time to network collapse over 40 simulations. (J) Schematic representing the proposed molecular mechanism underlying the stability of leading edge shape, with time increasing from i-iv.

169 Following a similar process of elimination, we determined that in fact only two elements were required

170 for stability. First, as reported previously for dendritic actin network polymerization against a single stiff

171 obstacle, it was necessary to constrain branching to occur only within a fixed distance from the leading

172 edge membrane (Fig. 2A-B) in order to maintain a steady state actin density (37). The molecular

173 motivation for this spatially limited "branching window" is rooted in that fact that activators of the Arp $2 / 3$

174 complex, which render Arp2/3 competent for actin filament nucleation, are typically membrane-associated

175 proteins (41). Second, we found that stability is inherently tied to the ability of filaments to spread laterally 
Garner and Theriot, 2020

176 to neighboring membrane segments (Fig. 2B ii-iii, Fig. 4F-I). (Recall that, because the branched actin 177 network geometry causes filaments to grow, on average, at an angle relative to the membrane normal (20, 178 21), polymerizing tips spread laterally along the leading edge $(16,19)$.) Removing filament spreading 179 from the model by fixing filaments to remain associated with their nearest membrane segment at birth 180 (Materials and Methods) led to actin density divergence: network regions with low filament density 181 eventually underwent complete depolymerization, while high-density regions continued to accumulate $182 \operatorname{actin}($ Fig. 4F-G).

These findings lead us to a simple molecular mechanism for leading edge stability, based on a 184 synergy between filament spreading and membrane-proximal branching (Fig. 4J): To begin with, regions 185 with initially high filament density come to protrude beyond the average position of the rest of the 186 membrane, representing the emergence of a leading edge shape fluctuation (Fig. 4J, $i$-ii). This induces 187 asymmetric filament spreading, where filaments from high-density regions can spread productively into 188 neighboring regions (Fig. 2B, $i$ ), but filaments spreading from adjacent low-density regions cannot keep 189 up with the fast moving membrane segments (Fig. 2B, iii), and thus are unproductive (Fig. 4J, ii-iii). In 190 this way, the branched geometry inherent to dendritic actin polymerization, as well as its interaction with 191 the shape of the membrane, naturally encodes leading edge stability (Fig. 4J, $i v$ ). Thus our results directly 192 demonstrate a "stability-through-spreading" mechanism that has previously only been assumed in mean193 field analytical theories (16). Remarkably, this means that leading edge maintenance is an intrinsic, 194 emergent property of branched actin network growth against a membrane, without requiring any further 195 regulatory governance. Geometrical constraints imposed simply by the nature of membrane-proximal 196 actin branching ensure that any small variations in either local actin filament density or growth rate are 197 inherently self-correcting to regress toward the mean. 
Garner and Theriot, 2020

Of note, it has previously been shown that Arp2/3-mediated branching is required for lamellipodial

formation in a wide variety of cell types; cells with inhibited or depleted Arp2/3 complex exhibit complete

disruption of the lamellipodium shape and often switch to a different mode of migration altogether, such

as filopodial motility $(14,42,43)$. Indeed, HL-60s treated with the Arp2/3 inhibitor CK-666 have

extremely variable leading edge shapes, characterized by long, thin filopodia-like protrusions (Movie S5).

Our theoretical results provide a mechanistic interpretation for this striking phenomenon, suggesting that

the vital lamellipodial maintenance role of Arp2/3-mediated branching stems from its ability to mediate

efficient filament spreading and equilibration of actin density fluctuations, purely because the daughter

filament always grows at an angle distinct from its mother.

\section{Optimal suppression of fluctuations by the highly-conserved $\sim 70^{\circ}$ branching angle}

Given the essential contribution of branched network geometry to the stability of the simulated leading edges, we reasoned that variations in the branching geometry alone might have a significant effect on leading edge fluctuations. We therefore performed simulations to determine the effects of changing the average branching angle and branching angle variability on filament orientation, filament density, and

leading edge fluctuation fit parameters (Fig. 5). In this context, we highlight the distinction between the branching angle, $\theta_{b r}$ (i.e., the angle of a daughter filament relative to its mother), and the filament angle or orientation, $\theta_{f}$ (i.e., the angle of a filament relative to the local membrane normal) (Fig. 5A, inset). In effect, our simulations represent selection assays, as mother filaments compete to stay within the fixed branching window, spawn daughter branches, and thus pass down their angle to their progeny (20). For example, when filaments are initialized with a random orientation, and the branching angle is fixed (i.e., there is no variability in the branching angle), only a handful of mother filament angles $\left(\theta_{f}\right)$ survive until the end of the simulation (Fig. 5A-C). The surviving, successful filament angles are narrowly and 
bioRxiv preprint doi: https://doi.org/10.1101/2020.08.22.262907; this version posted August 22, 2020. The copyright holder for this preprint (which was not certified by peer review) is the author/funder, who has granted bioRxiv a license to display the preprint in perpetuity. It is made available under aCC-BY-NC-ND 4.0 International license.

Garner and Theriot, 2020

A

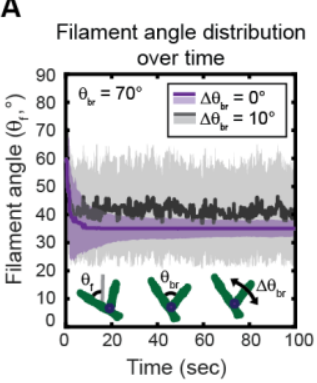

$\mathbf{F}$

Simulated leading edges: Fitted amplitude

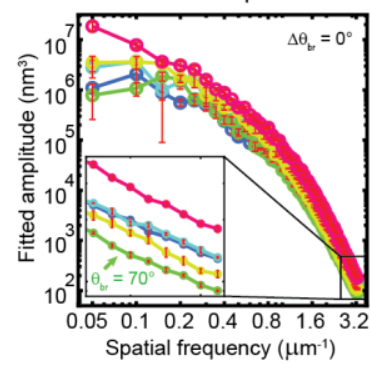

J

Simulated leading edges: Fitted amplitude

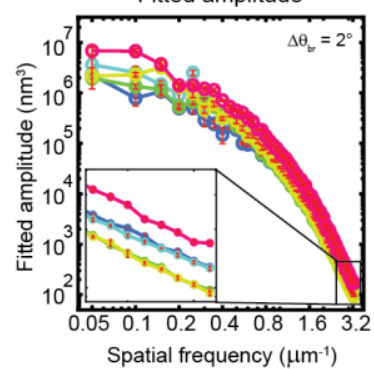

$\mathbf{N}$

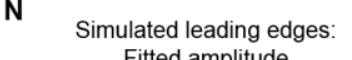
Fitted amplitude

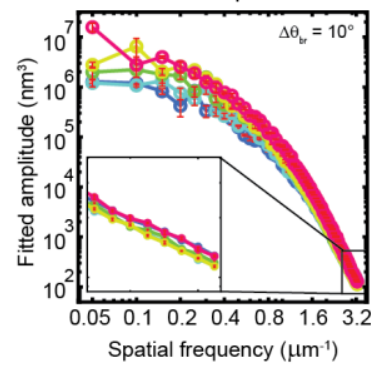

B

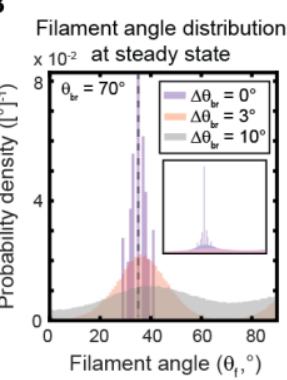

G

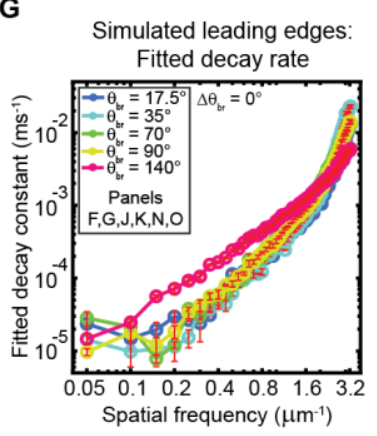

$\mathrm{K}$

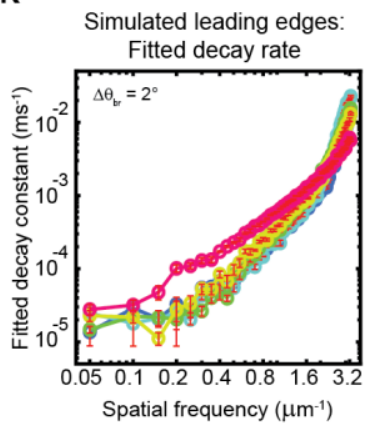

0

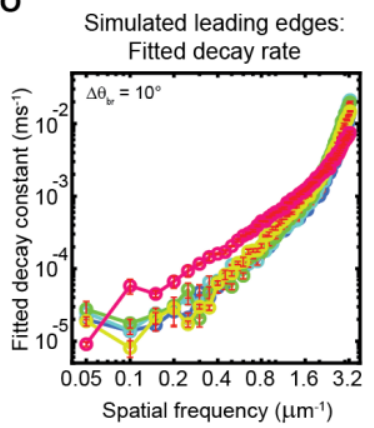

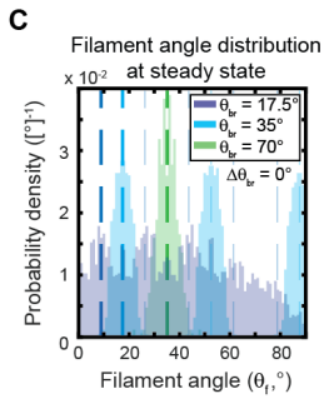

D

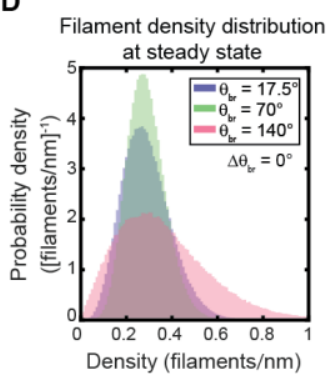

H

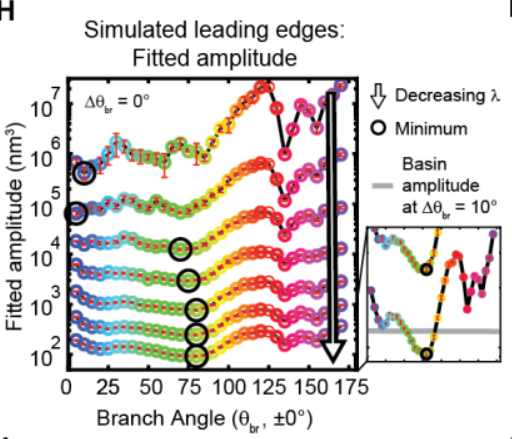

L

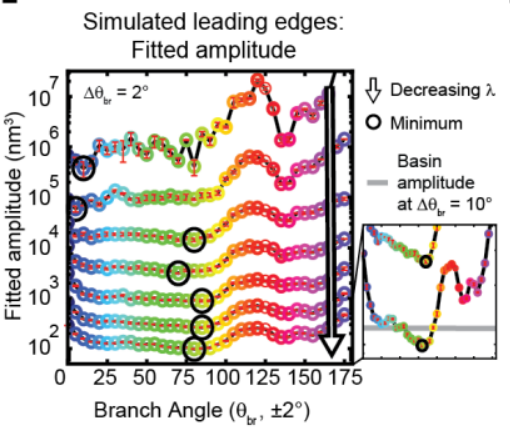

$\mathbf{P}$

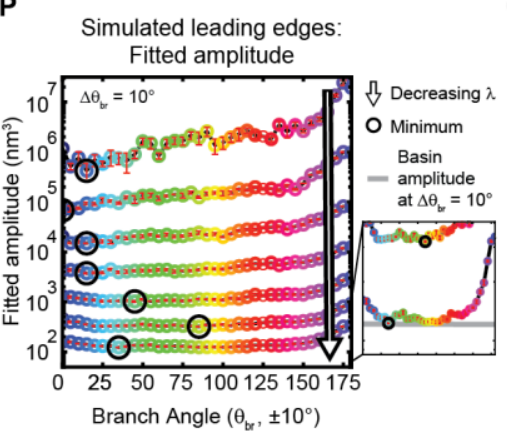

E

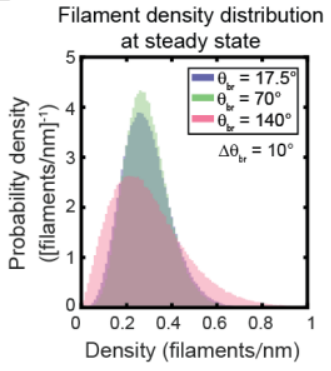

I

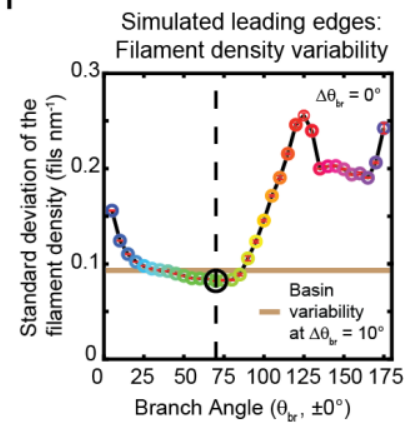

M

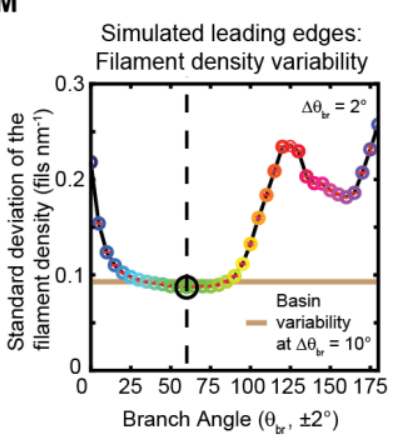

Q

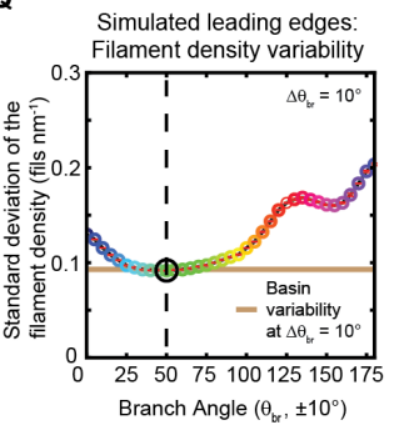

Fig. 5. The genetically-encoded Arp2/3-mediated branching angle is optimal for suppressing leading edge fluctuations. (A-C) Time course (A) and steady state distribution (B-C) of the filament angle $\left(\theta_{\mathrm{f}}\right)$ for simulations with various branching angle standard deviations $\left(\Delta \theta_{\mathrm{br}}, \mathrm{A}-\mathrm{B}\right)$ and means $\left(\theta_{\mathrm{br}}, \mathrm{C}\right)$. Dashed lines represent $\theta_{\mathrm{br}} / 2$ plus integer multiples of $\theta_{\mathrm{br}}$. (D-E) Steady state filament density distribution as a function of the mean branching angle in the context of $\Delta \theta_{\mathrm{br}}=0^{\circ}$ (D) and $\Delta \theta_{\mathrm{br}}=10^{\circ}$ (E). (A-B, D-E) Results from a representative simulation for each condition. (C) Data integrated over 40 simulations. (F-Q) Predicted response of leading edge fluctuations and filament density variability to changes in the branch angle and branch angle variability, (continued on next page) 
Garner and Theriot, 2020

(Fig. 5 continued) medians over 40 simulations for each condition. Red error bars - standard error. Color map is identical for panels C-Q. (H,L,P) Fitted amplitude as a function of branch angle, where each line represents a different spatial frequency, increasing from 0.2-3.2 um $\mathrm{um}^{-1}$ in intervals of $0.5 \mathrm{um}^{-}$ ${ }^{1}$. Insets have identical $x$-axes to main panels. (I,M,Q) Standard deviation of the filament density at steady state plotted as a function of branch angle. Note the $\mathrm{x}$ - and $\mathrm{y}$-axes limits in (F-G, J-K, N-O) are expanded compared to the equivalent panels in Figs. 1-3.

symmetrically distributed around one half of the branching angle (Fig. 5B-C). This optimal filament angle allows mother and daughter filaments to branch back and forth symmetrically about the membrane normal, such that mother filaments do not out-compete their progeny (Fig. 2B) (20).

In living cells, branching is mediated by the Arp $2 / 3$ complex, which has been experimentally measured to form highly regular and sterotyped branches at $\sim 70^{\circ}(22-24)$. Intriguingly, this protein complex is highly conserved (44), with measurements of the branching angle in a wide variety of species (protists $(22-24,45)$, yeast (24), mammals $(24,45,46)$, and amphibians (47)) using various experimental techniques (platinum replica electron microscopy, cryo-electron microscopy, and total internal reflection microscopy) all falling within the range of $67-78^{\circ}\left( \pm 2-13^{\circ}\right)$. The high degree of conservation hints that this specific angle might carry some functional optimality, but the question has not been addressable experimentally; due to the lack of naturally occuring Arp2/3 variants with a substantially different branching angle, an alternative branching structure would hypothetically have to be designed do novo, presumably by altering the protein interaction interface by which the Arp $2 / 3$ complex binds to the side of a mother filament (23). We thus sought to explore the possible functional significance of this conserved angle using our minimal stochastic model. Excitingly, we found that in simulations with no branch angle variability, a $70-80^{\circ}$ branching angle was optimal for minimizing both actin density fluctuations (Fig. 5D,I) and leading edge fluctuation amplitudes for wavelengths smaller than $\sim 2 \mu \mathrm{m}$ (Fig. 5F,H). These results provide tantalizing mechanistic insight into the long-standing question of why this angle is so ubiquitous. 
Garner and Theriot, 2020

242 the orientation of the daughters) is set by the degree of variability in the branching angle (which, in turn, 243 reflects the influence of thermal fluctuations). Perhaps unsurprisingly, decreasing this orientational 244 heritability significantly reduces the dependence of fluctuation amplitude and filament density variability 245 on the branching angle (Fig. 5J-Q), and thereby counteracts the beneficial effect of the optimal angle on 246 leading edge fluctuations. Introducing a branch angle variability of $\pm 2^{\circ}$ (on the lower end of the 247 experimentally-measured values) broadens the range of near-optimal branch angles but maintains the 248 optimum at $\sim 70-80^{\circ}$ (Fig. 5J,L), while introducing a variability of $\pm 10^{\circ}$ (on the higher end of the measured 249 range) completely removes the optimum (Fig. 5N,P). In both cases, increasing the branch angle variability 250 increases the minimum possible fluctuation amplitude (Fig. 5H,L,P - insets) and filament density 251 variability (Fig. 5I,M,Q), representing a decrease in the noise-suppression capabilities of the system. 252 Overall, these results provide strong support for the idea that actin network geometry is not only essential 253 for leading edge stability, but also plays a major role in determining the fundamental limits of smoothness 254 in lamellipodial shape.

\section{Discussion}

257 The emergence of robust collective behaviors from stochastic elements is an enduring biological mystery 258 which we are only beginning to unravel (48-54). The apparent dichotomy in actin-based motility between 259 the random elongation of individual filaments and the stable formation of smooth and persistent higher260 order actin structures such as lamellipodia exemplifies this enigma, and provides an avenue towards 261 understanding general strategies for noise suppression in biological systems. In recent years it has become clear that perturbation-free experiments which examine fluctuations 263 around the mean at steady state (in contrast to probing the change in the mean due to a perturbation) can 
Garner and Theriot, 2020

264 be a powerful tool for understanding noisy systems (55). In this work, application of that principle in 265 combination with high-precision measurements, quantitative analytical techniques, and physical modeling 266 led to the surprising revelation that the suppression of stochastic fluctuations naturally emerges from the 267 interactions between a growing actin network and the leading edge membrane, with no additional 268 feedback required.

\section{3} computational model might be useful for exploring the effects of various extracellular forces, such as those 276 produced by obstacles or variations in matrix density. We also note that a certain degree of biochemical molecules (64). How local biochemical control (or lack thereof) over these rate constants might affect leading edge fluctuations remains an interesting avenue for future investigation, both theoretically and experimentally.

284 leading edge. By selectively removing elements of the model, we determined that lateral filament 285 spreading, combined with a fixed branching window, is indispensable for leading edge stability. This 286 highlights the crucial role in lamellipodial maintenance of the branched structure of actin networks, 
287 wherein each daughter filament inherits angular information from its mother. Our further investigations 288 into the evolutionary properties of actin network growth revealed that a $\sim 70-80^{\circ}$ branch angle maximally 289 suppresses fine-scale actin density and leading edge shape fluctuations, showing for the first time that 290 Arp2/3-mediated branching imparts optimal functionality, as was long hypothesized based on strong 291 sequence, structural, and functional conservation throughout the eukaryotic tree of life.

292 Returning to the broader question of how noisy biological systems control for stochasticity, we 293 find that stability in the case of lamellipodial dynamics is inherently encoded by the geometry of branched 294 actin network growth. It will be interesting to see whether similar principles hold for other cytoskeletal 295 structures with clear geometric constraints, such as endocytic pits, the cytokinetic ring, and the mitotic 296 spindle. 
Garner and Theriot, 2020

\section{References:}

1. T. D. Pollard, J. A. Cooper, Actin, a Central Player in Cell Shape and Movement. Science. 326, $1208-1212$ (2009).

2. L. K. Fritz-Laylin, S. J. Lord, R. D. Mullins, WASP and SCAR are evolutionarily conserved in actin-filled pseudopod-based motility. J. Cell Biol. 216, 1673-1688 (2017).

3. T. Svitkina, The actin cytoskeleton and actin-based motility. Cold Spring Harb. Perspect. Biol. 10, 1-21 (2018).

4. A. Mogilner, G. Oster, Cell motility driven by actin polymerization. Biophys. J. 71, 3030-45 (1996).

5. C. S. Peskin, G. M. Odell, G. F. Oster, Cellular motions and thermal fluctuations: the Brownian ratchet. Biophys. J. 65, 316-324 (1993).

6. J. A. Theriot, T. J. Mitchison, L. G. Tilney, D. A. Portnoy, The rate of actin-based motility of intracellular Listeria monocytogenes equals the rate of actin polymerization. Nature. 357, 257260 (1992).

7. M. Prass, K. Jacobson, A. Mogilner, M. Radmacher, Direct measurement of the lamellipodial protrusive force in a migrating cell. J. Cell Biol. 174, 767-772 (2006).

8. T. M. Svitkina, Ultrastructure of protrusive actin filament arrays. Curr. Opin. Cell Biol. 25, 574$581(2013)$

9. S. M. Rafelski, J. A. Theriot, Crawling Toward a Unified Model of Cell Motility: Spatial and Temporal Regulation of Actin Dynamics. Annu. Rev. Biochem. 73, 209-239 (2004).

10. D. Vavylonis, Q. Yang, B. O’Shaughnessy, Actin polymerization kinetics, cap structure, and fluctuations. Proc. Natl. Acad. Sci. U. S. A. 102, 8543-8548 (2005).

11. T. M. Svitkina, A. B. Verkhovsky, K. M. McQuade, G. G. Borisy, Analysis of the actin-myosin II 
Garner and Theriot, 2020

system in fish epidermal keratocytes: Mechanism of cell body translocation. J. Cell Biol. 139, 397-415 (1997).

12. V. C. Abraham, V. Krishnamurthi, D. L. Taylor, F. Lanni, The Actin-Based Nanomachine at the Leading Edge of Migrating Cells. Biophys. J. 77, 1721-1732 (1999).

13. V. M. Laurent, S. Kasas, A. Yersin, T. E. Schäffer, S. Catsicas, G. Dietler, A. B. Verkhovsky, J.J. Meister, Gradient of rigidity in the lamellipodia of migrating cells revealed by atomic force

14. L. K. Fritz-Laylin, M. Riel-Mehan, B.-C. Chen, S. J. Lord, T. D. Goddard, T. E. Ferrin, S. M.

Nicholson-Dykstra, H. Higgs, G. T. Johnson, E. Betzig, R. D. Mullins, Actin-based protrusions of

15. T. Y. C. Tsai, S. R. Collins, C. K. Chan, A. Hadjitheodorou, P.-Y. Lam, S. S. Lou, H. W. Yang, J.

Jorgensen, F. Ellett, D. Irimia, M. W. Davidson, R. S. Fischer, A. Huttenlocher, T. Meyer, J. E.

Ferrell, J. A. Theriot, Efficient Front-Rear Coupling in Neutrophil Chemotaxis by Dynamic

Myosin II Localization. Dev. Cell. 49, 189-205 (2019). Actin Filament Growth Dynamics. PLoS Biol. 5, 2035-2052 (2007).

17. S. De Oliveira, E. E. Rosowski, A. Huttenlocher, Neutrophil migration in infection and wound repair: Going forward in reverse. Nat. Rev. Immunol. 16, 378-391 (2016).

18. E. Kolaczkowska, P. Kubes, Neutrophil recruitment and function in health and inflammation. Nat. Rev. Immunol. 13, 159-175 (2013).

9. H. P. Grimm, A. B. Verkhovsky, A. Mogilner, J. J. Meister, Analysis of actin dynamics at the 
Garner and Theriot, 2020

leading edge of crawling cells: implications for the shape of keratocyte lamellipodia. Eur.

Biophys. J. 32, 563-577 (2003).

20. I. V. Maly, G. G. Borisy, Self-organization of a propulsive actin network as an evolutionary process. Proc. Natl. Acad. Sci. 98, 11324-11329 (2001).

21. A. B. Verkhovsky, O. Y. Chaga, S. Schaub, T. M. Svitkina, J.-J. Meister, G. G. Borisy,

Orientational Order of the Lamellipodial Actin Network as Demonstrated in Living Motile Cells.

Mol. Biol. Cell. 14, 2559-2569 (2003).

22. R. D. Mullins, J. A. Heuser, T. D. Pollard, The interaction of Arp2/3 complex with actin:

Nucleation, high affinity pointed end capping, and formation of branching networks of filaments.

Proc. Natl. Acad. Sci. 95, 6181-6186 (1998).

23. N. Volkmann, K. J. Amann, S. Stoilova-McPhie, C. Egile, D. C. Winter, L. Hazelwood, J. E.

Heuser, R. Li, T. D. Pollard, D. Hanein, Structure of arp2/3 complex in its activated state and in actin filament branch junctions. Science. 293, 2456-2459 (2001).

24. I. Rouiller, X. P. Xu, K. J. Amann, C. Egile, S. Nickell, D. Nicastro, R. Li, T. D. Pollard, N.

Volkmann, D. Hanein, The structural basis of actin filament branching by the Arp2/3 complex. $J$.

Cell Biol. 180, 887-895 (2008).

25. A. Diz-Muñoz, K. Thurley, S. Chintamen, S. J. Altschuler, L. F. Wu, D. A. Fletcher, O. D. Weiner, Membrane Tension Acts Through PLD2 and mTORC2 to Limit Actin Network Assembly During Neutrophil Migration. PLOS Biol. 14, e1002474 (2016). signals to the leading edge during neutrophil migration. Cell. 148, 175-188 (2012).

27. J. Mueller, G. Szep, M. Nemethova, I. de Vries, A. D. Lieber, C. Winkler, K. Kruse, J. V. Small, 
Garner and Theriot, 2020

366

367

368

369

370

371

372

373

374

375

376

377

378

379

380

381

382

383

384

385

386

387

388

C. Schmeiser, K. Keren, R. Hauschild, M. Sixt, Load Adaptation of Lamellipodial Actin Networks. Cell. 171, 188-200 (2017).

28. N. C. Gauthier, T. A. Masters, M. P. Sheetz, Mechanical feedback between membrane tension and dynamics. Trends Cell Biol. 22, 527-535 (2012).

29. K. Tsujita, T. Takenawa, T. Itoh, Feedback regulation between plasma membrane tension and membrane-bending proteins organizes cell polarity during leading edge formation. Nat. Cell Biol. 17, 749-758 (2015).

30. V. I. Risca, E. B. Wang, O. Chaudhuri, J. J. Chia, P. L. Geissler, D. a. Fletcher, Actin filament curvature biases branching direction. Proc. Natl. Acad. Sci. 109, 2913-2918 (2012).

31. B. J. Spellberg, M. Collins, S. W. French, J. E. Edwards, Y. Fu, A. S. Ibrahim, A phagocytic cell line markedly improves survival of infected neutropenic mice. J. Leukoc. Biol. 78, 338-344 (2005).

32. A. Millius, O. D. Weiner, in Chemotaxis (Humana Press, Totowa, NJ, 2009; http://link.springer.com/10.1007/978-1-60761-198-1_11), vol. 571, pp. 167-177.

33. Neutrophil-like HL-60 cells expressing only GFP-tagged $\beta$-actin exhibit nearly normal motility, doi:https://doi.org/10.1002/cm.21603.

34. F. L. H. Brown, Elastic Modeling of Biomembranes and Lipid Bilayers. Annu. Rev. Phys. Chem. 59, 685-712 (2008).

35. P. G. De Gennes, Dynamics of Entangled Polymer Solutions. I. The Rouse Model. Macromolecules. 9, 587-593 (1976).

36. E. Cerda, L. Mahadevan, Geometry and Physics of Wrinkling. Phys. Rev. Lett. 90, 074302 (2003).

37. A. E. Carlsson, Growth of Branched Actin Networks against Obstacles. Biophys. J. 81, 1907- 
Garner and Theriot, 2020

$1923(2001)$.

38. A. E. Carlsson, Growth Velocities of Branched Actin Networks. Biophys. J. 84, 2907-2918 (2003).

39. A. Bisaria, A. Hayer, D. Garbett, D. Cohen, T. Meyer, Membrane-proximal F-actin restricts local membrane protrusions and directs cell migration. Science. 368, 1205-1210 (2020).

41. S. Suetsugu, Activation of nucleation promoting factors for directional actin filament elongation: Allosteric regulation and multimerization on the membrane. Semin. Cell Dev. Biol. 24, 267-271 (2013).

42. J. H. Henson, M. Yeterian, R. M. Weeks, a. E. Medrano, B. L. Brown, H. L. Geist, M. D. Pais, R. Oldenbourg, C. B. Shuster, Arp2/3 Complex Inhibition Radically Alters Lamellipodial Actin Architecture, Suspended Cell Shape, and the Cell Spreading Process. Mol. Biol. Cell. 26 (2015), doi:10.1091/mbc.E14-07-1244.

43. C. Wu, S. B. Asokan, M. E. Berginski, E. M. Haynes, N. E. Sharpless, J. D. Griffith, S. M. Gomez, J. E. Bear, Arp2/3 is critical for lamellipodia and response to extracellular matrix cues but is dispensable for chemotaxis. Cell. 148, 973-987 (2012).

44. M. D. Welch, A. H. Depace, S. Verma, A. Iwamatsu, T. J. Mitchison, The Human Arp2/3 Complex Is Composed of Evolutionarily Conserved Subunits and Is Localized to Cellular Regions of Dynamic Actin Filament Assembly. J. Cell Biol. 138, 375-384 (1997).

45. L. Blanchoin, K. J. Amann, H. N. Higgs, J. Marchand, D. A. Kaiser, T. D. Pollard, Direct observation of dendritic actin filament networks nucleated by Arp2/3 complex and WASP/Scar proteins. Nature. 404, 1007-1011 (2000). 
Garner and Theriot, 2020

46. L. Cai, A. M. Makhov, D. A. Schafer, J. E. Bear, Coronin 1B antagonizes cortactin and remodels Arp2/3-containing actin branches in lamellipodia. Cell. 134, 828-842 (2008).

47. T. M. Svitkina, G. G. Borisy, Arp2/3 complex and actin depolymerizing factor/cofilin in dendritic organization and treadmilling of actin filament array in lamellipodia. J. Cell Biol. 145, 1009-1026 (1999).

48. W. Y. C. Huang, Q. Yan, W. C. Lin, J. K. Chung, S. D. Hansen, S. M. Christensen, H. L. Tu, J. Kuriyan, J. T. Groves, Phosphotyrosine-mediated LAT assembly on membranes drives kinetic bifurcation in recruitment dynamics of the Ras activator SOS. Proc. Natl. Acad. Sci. 113, 82188223 (2016).

49. N. Battich, T. Stoeger, L. Pelkmans, Control of Transcript Variability in Single Mammalian Cells. Cell. 163, 1596-1610 (2015).

50. A. Y. Chang, W. F. Marshall, Organelles - Understanding noise and heterogeneity in cell biology at an intermediate scale. J. Cell Sci. 130, 819-826 (2017).

51. L. Mohapatra, B. L. Goode, P. Jelenkovic, R. Phillips, J. Kondev, Design Principles of Length Control of Cytoskeletal Structures. Annu. Rev. Biophys. 45, 85-116 (2016).

52. A. Raj, A. van Oudenaarden, Nature, Nurture, or Chance: Stochastic Gene Expression and Its Consequences. Cell. 135, 216-226 (2008).

53. W. T. Gray, S. K. Govers, Y. Xiang, B. R. Parry, M. Campos, S. Kim, C. Jacobs-Wagner, Nucleoid Size Scaling and Intracellular Organization of Translation across Bacteria. Cell. 177, 1632-1648.e20 (2019).

54. A. C. Oates, What's all the noise about developmental stochasticity? Development. 138, 601-607 (2011).

55. E. S. Welf, G. Danuser, Using fluctuation analysis to establish causal relations between cellular 
Garner and Theriot, 2020

events without experimental perturbation. Biophys. J. 107, 2492-2498 (2014).

436 56. A. Mogilner, G. Oster, Force generation by actin polymerization II: The elastic ratchet and tethered filaments. Biophys. J. 84, 1591-1605 (2003).

58. J. B. Alberts, G. M. Odell, In silico reconstitution of Listeria propulsion exhibits nano-saltation. PLoS Biol. 2 (2004), doi:10.1371/journal.pbio.0020412.

59. S. C. Kuo, J. L. McGrath, Steps and fluctuations of Listeria monocytogenes during actin-based motility. Nature. 407, 1026-1029 (2000).

60. R. D. Mullins, P. Bieling, D. A. Fletcher, From solution to surface to filament: actin flux into branched networks. Biophys. Rev. 10, 1537-1551 (2018).

61. O. Chaudhuri, S. H. Parekh, D. A. Fletcher, Reversible stress softening of actin networks. Nature. 445, 295-298 (2007).

64. P. N. Devreotes, S. Bhattacharya, M. Edwards, P. A. Iglesias, T. Lampert, Y. Miao, Excitable 
Garner and Theriot, 2020

\section{Acknowledgements:}

457 We kindly thank E. F. Koslover and A. J. Spakowitz for their helpful advice on model conceptualization 458 and data analysis, and E. F. Koslover, N. M. Belliveau, P. Radhakrishnan, and A. Savinov for helpful 459 comments on the manuscript. Funding: Howard Hughes Medical Institute, Washington Research 460 Foundation, NIH Grant R37-AI036929 to J.A.T., Gerald J. Lieberman Fellowship to R.M.G, NSF 461 Graduate Research Fellowship to R.M.G.

462

\section{Author contributions:}

464 Study was conceived by R.M.G. and J.A.T. All experiments, modeling, and analysis were carried out by 465 R.M.G. Interpretation of the results and preparation of the manuscript were performed by R.M.G. and 466 J.A.T.

468 Competing Interests Statement:

469 The authors declare no competing financial interests.

471 Data and Materials Availability:

472 Analysis and modeling code for this paper is available on the Theriot lab Gitlab:

$473<\underline{\text { https://gitlab.com/theriot_lab/leading-edge-stability-in-motile-cells-is-an-emergent-property-of- }}$

474 branched-actin-network-growth $>$. All data will be made available upon reasonable request to the 475 corresponding author. 